\title{
EDITORIAL
}

\section{Insufficient Scientific Evidence Supporting the Use of Ivermectin in the Prevention and Treatment of COVID-19}

Ivermectin- a drug obtained following fermentation of Streptomyces avermitilis, was discovered in 1975 and was approved for medical use in 1981. Its discovery was hailed as an innovation in medicine resulting in the conferring of the 2015 Nobel Prize in Physiology or Medicine to William Campbell and Satoshi Ōmura for its discovery and applications. In humans, U.S. Food and Drug Administration (FDA) has approved Ivermectin for the treatment of parasite infestations which includes head lice, scabies, river blindness, lymphatic filariasis, strongyloidiasis, trichuriasis and ascariasis. In veterinary medicine, Ivermectin is used to prevent and treat heartworm and acariasis, among other diseases.

The anti-parasitic mode of action of Ivermectin has been well-defined. It acts by binding to the Gammaaminobutyric acid (GABA) receptors which results in increased influx of Chloride ion with consequential hyperpolarization and paralysis. On the contrary, its mode of action in SARS-CoV-2 infection is debatable. While the conclusions of this manuscript are currently under reconsideration and are subject to serious scientific criticism, Zaidi and Dehgani-Mobaraki have suggested that in addition to inhibiting host importin alpha/beta-1 nuclear transport proteins, which are part of a key intracellular transport process that viruses hijack to enhance infection by suppressing the host's antiviral response, Ivermectin also acts by inhibiting the binding of SARS-CoV-2 to ACE-2 receptors (1). A recent study showed Ivermectin had proven effective as an inhibitor of COVID-19, however the experiments conducted in the study were in vitro and the trials had yet to be conducted in humans (2).

Despite these contradictory observations and in the face of the lack of an approved drug treatment for COVID-19, many patients resorted to the use of Ivermectin for both the prevention and the treatment of infection with SARS-CoV-2. An extremely controversial manuscript that was initially published in January 2021 and subsequently withdrawn by the editors of the Frontiers in Pharmacology had undesirable consequences (3). The editors concluded that this manuscript contained unsubstantiated claims and violated the journal's editorial policies. Unfortunately, despite of its withdrawal, this manuscript was used by many providers to justify the use of Ivermectin for the prevention and treatment of COVID-19. In an independent review of available literature, the author's concluded that "Based on the current very low- to low-certainty evidence, we are uncertain about the efficacy and safety of Ivermectin used to treat or prevent COVID-19. The completed studies are small, and few are considered high quality. Several studies are underway that may produce clearer answers in review updates. Overall, the reliable evidence available does not support the use of Ivermectin for treatment or prevention of COVID-19 outside of well-designed randomized trials" (4).

Regrettably, despite overwhelming scientific evidence denouncing the use of Ivermectin for prevention and treatment of COVID-19 supported by strong FDA denunciation, the use of this drug for this indication has not abated (5). A recent study examining trends in Ivermectin dispensing from outpatient retail pharmacies in the United States during the COVID-19 pandemic showed an increase from an average of 3,600 prescriptions per week at the pre-pandemic baseline (March 16, 2019-March 13, 2020) to a peak of 39,000 prescriptions in the week ending on January 8, 2021. Since early July 2021, outpatient Ivermectin dispensing has again begun to rapidly increase, reaching more than 88,000 prescriptions in the week ending August 13, 2021. This represents a 24fold increase from the pre-pandemic baseline. (6). 
The recent use of Ivermectin that has been formulated for veterinary use has created additional challenges. Self-administering of the drug for SARS-CoV-2 infections have led to an unprecedented increase in calls to the Texas Poison Center Network. The Texas Department of State Health Services has issued a health advisory over the improper use of Ivermectin to treat or prevent COVID-19 after calls to the Texas Poison Center Network for people exposed to the drug increased $150 \%$ (7). This is a nation-wide observation as most hospitals have reported similar toxicity profiles due to iniquitous use of Ivermectin for the prevention and treatment of COVID- 19 .

In addition to the FDA and the U.S. Centers for Disease Control and Prevention, organizations such as American Medical Association and American Pharmacist Association have also strongly opposed the ordering, prescribing, or dispensing of Ivermectin to prevent or treat COVID-19 outside of a clinical trial (8). National Institutes of Health has designed double-blinded randomized placebocontrol clinical trials to ascertain the safety and effectiveness of Ivermectin and other repurposed drugs in the prevention and treatment of COVID19. DHR Health Institute for Research and Development is involved in these studies, and it is expected that the results of these prospective clinical trials would unequivocally establish the benefit (or otherwise) of these agents.

Considering the available scientific evidence, it is strongly recommended that Ivermectin should not be prescribed and/or self-administered for the prevention or treatment of COVID-19. Additionally, it must be stressed that vaccination remains the most effective approach for the prevention of COVID-19 and informing the public about proper treatment options is the apposite course of action towards ending this global pandemic.

\section{References}

1. Zaidi, A.K., Dehgani-Mobaraki, P. The mechanisms of action of Ivermectin against SARS-CoV-2: An evidence-based clinical review article. J Antibiot (2021). https://doi.org/10.1038/s41429-021-00430-5

2. Caly, L., Druce, J.D., et.al. The FDA-approved drug Ivermectin inhibits the replication of SARS-CoV-2 in vitro. Antiviral Res. 2020; 178:104787.
3. Offord, C. Frontiers Pulls Special COVID-19 Issue After Content Dispute. The Scientist. 2021. https://www.thescientist.com/news-opinion/frontiers-pulls-special-covid-19issue-after-content-dispute-68721

4. Popp, M., Stegemann, M., et. al. Ivermectin for preventing and treating COVID-19. Cochrane Database of Systematic Reviews 2021, Issue 7. Art, No: CD015017. DO1: https://www.cochranelibrary.com/cdsr/doi/10.1002/14651858. CD015017.pub2/full

5. Why You Should Not Use Ivermectin to Treat or Prevent COVID-19. 2021. https://www.fda.gov/consumers/consumerupdates/why-you-should-not-use-ivermectin-treat-or-preventcovid-19

6. U.S. Centers for Disease Control \& Prevention. Emergency Preparedness and Response. Rapid Increase in Ivermectin Prescriptions and Reports of Severe Illness Associated with Use of Products Containing Ivermectin to Prevent or Treat COVID19.

2021.

https://emergency.cdc.gov/han/2021/han00449.asp?ACSTracki ngID=USCDC 511 -

DM64535\&ACSTrackingLabel=HAN\%20449\%20$\% 20$ General $\% 20$ Public\&deliveryName=USCDC 511 DM64535

7. Texas Poison Center Calls Triple for People Treating COVID19 With Ivermectin. 2021. https://www.msn.com/enus/health/medical/texas-poison-center-calls-triple-for-peopleself-treating-covid-19-with-ivermectin/ar-AANP5wz

8. AMA, APhA, ASHP statement on ending use of Ivermectin to treat COVID-19. 2021. https://www.ama-assn.org/presscenter/press-releases/ama-apha-ashp-statement-ending-useivermectin-treat-covid-19

\section{Sohail Rao, MD, MA, DPhil}

Executive Vice President, DHR Health, 5501 S. McColl Road, Edinburg, Texas

President \& Chief Executive Officer, DHR Health Institute for Research \& Development, 5323 S. McColl Road, Edinburg, Texas

Corresponding author email: s.rao@dhr-rgv.com

Disclosures: None

ORCID: Sohail Rao: https://orcid.org/0000-0001-50279992

Alec Vasquez-Kanhere

DHR Health Institute for Research \& Development, 5323 S. McColl Road, Edinburg, Texas

Disclosures: None

ORCID: Alec Vasquez-Kanhere:

https://orcid.org/0000-0002-2566-1724 


\section{Manish Singh, MD, FACS}

Chief Executive Officer, DHR Health, 5501 S. McColl Road, Edinburg, Texas

Disclosures: None

ORCID: Manish Singh: https://orcid.org/0000-00034146-3282 